\title{
Semiclassical Approximation to Neutron Star Superfluidity Corrected for Proximity Effects
}

\author{
F. Barranco ${ }^{a}$, R.A. Broglia ${ }^{b, c}$, H. Esbensen ${ }^{d}$ and E. Vigezzi $^{b}$ \\ ${ }^{a}$ Dpto. de Física Aplicada, Escuela Superior de Ingenieros, Universidad de Sevilla, Spain. \\ ${ }^{b}$ Dipartimento di Fisica, Università di Milano and INFN, Sezione di Milano, Italy. \\ ${ }^{c}$ The Niels Bohr Institute, University of Copenhagen, Denmark. \\ ${ }^{d}$ Physics Division, Argonne National Laboratory, Argonne, Illinois 60439, USA.
}

(May 11, 2018)

\begin{abstract}
The inner crust of a neutron star is a superfluid and inhomogeneous system, consisting of a lattice of nuclei immersed in a sea of neutrons. We perform a quantum calculation of the associated pairing gap and compare it to the results one obtains in the Local Density Approximation (LDA). It is found that the LDA overestimates the spatial dependence of the gap, and leads to a specific heat of the system which is too large at low temperatures, as compared with the quantal result. This is caused by the neglect of proximity effects and the delocalized character of the single-particle wavefunctions close to the Fermi energy. It is possible to introduce an alternative, simple semiclassical approximation of the pairing gap which leads to a specific heat that is in good agreement with the quantum calculation.
\end{abstract}

PACS numbers: 21.60.-n, 26.60.+c, 97.60.Jd

keywords: pairing gap, neutron stars 


\section{INTRODUCTION}

Above a density of about about $3 \times 10^{11} \mathrm{~g} \mathrm{~cm}^{-3}$ (corresponding to $1.8 \times 10^{-4} \mathrm{n} \mathrm{fm}^{-3}$ ) matter in neutron stars consists of a matrix of nuclei immersed in a sea of neutrons and an approximately uniform sea of electrons. Such a configuration persists up to roughly half nuclear saturation density, and constitutes the so-called "inner crust" of a neutron star. At low temperatures, this system is superfluid with a positive Fermi energy $\epsilon_{F}$, and one is confronted with the task of estimating the pairing gap $\Delta$ of nuclei immersed in the neutron liquid [4]. With the exception of ref. [2], the pairing gap in this inhomogeneous medium has been calculated assuming that locally it coincides with the value of the pairing gap in bulk matter at the corresponding density (cf. e.g. ref. [3] and refs. therein).

A complete calculation of pairing should include the induced interaction, taking into account polarization effects in the medium (cf. for example [4). In the present paper we

will restrict ourselves to the mean field level, and we shall compare the results of a local density approximation (LDA) calculation of the pairing gap to the results obtained from a Hartree-Fock-Bogoliubov (HFB) calculation. We find that the LDA leads to a spatial variation of the gap near the surface of a nucleus which is stronger than that obtained in the HFB calculation. This is because a Cooper pair in the quantum (HFB) calculation samples the pairing interaction over a relatively large distance, which is of the order of the coherence length (cf. Section III). As a result, the pairing interaction in the neutron liquid influences the gap in nuclei, and vice versa, so that the spatial dependence of the gap is smeared out near the nuclear surface.

The pairing gap plays an important role in the thermal evolution of a neutron star, because it influences in an essential way the specific heat in the inner crust. We will show that the LDA overestimates the effect of the presence of nuclei on the specific heat by a large amount (cf. Section IV). In the LDA, the specific heat is obtained from an integral in phase space, and it is possible to single out two contributions to the specific heat, one from the interior of the nucleus and one from the outer neutron liquid. Inside the nucleus the local 
pairing gap is small, and this enhances the value of the specific heat. On the other hand, in the quantal calculation the specific heat is determined from the value of the pairing gap of the states close to the Fermi surface, which is obtained from an integral over the whole Wigner cell, and is much less sensitive to the presence of the nucleus. 


\section{PAIRING FIELD IN COORDINATE SPACE}

We consider a spherically symmetric system which is governed by a single-particle Hamiltonian $h$ and a two-body interaction $v$. The Hamiltonian $h=T+U$ consists of the kinetic energy and a mean-field $U$ which we do not calculate self-consistently but parametrize as described later on. The explicit effect of the two-body interaction on the ground state of the system is therefore included in the HFB equations only via the pairing field.

The system is placed into a spherical box with a nucleus at the origin. The radius of the box is equal to that of the Wigner-Seitz cell of the lattice (cf. Fig. 1). Nuclei belonging to different cells are so far away from each other, that one can solve for a single cell separately. The single-particle wavefunctions, which are eigenfunctions to $h$ are therefore required to

vanish at the boundary of the cell. They are denoted by $\phi_{n l j m}(\vec{r})=\vec{r} \mid n l j m>$, while $\epsilon_{n l j}$ are the associated eigenvalues.

The HFB equations [5] are solved in matrix form using this basis. We shall, in particular, make use of the two-particle wavefunctions

$$
<\vec{r}_{1} \vec{r}_{2}\left|n n^{\prime} l j, J=0>=\phi_{n l j}\left(r_{1}\right) \phi_{n^{\prime} l j}\left(r_{2}\right) \sum_{m} \frac{(-1)^{l+j-m} \sqrt{2}}{2 j+1}<\hat{r}_{1}\right| l j m><\hat{r}_{2} \mid l j-m>,
$$

where $J$ is the total angular momentum of the system and $\phi_{n l j}$ is a radial wavefunction. The normalization of these two-particle wavefunctions is $2 /(2 j+1)$.

In the case of spherical symmetry, the HFB equations are diagonal in the quantum numbers $(l j)$, having the form

$$
\begin{gathered}
\left(\epsilon_{n l j}-\epsilon_{F}\right) U_{n l j}^{q}+\sum_{n^{\prime}} \Delta_{n n^{\prime} l j} V_{n^{\prime} l j}^{q}=E_{q l j} U_{n l j}^{q}, \\
-\left(\epsilon_{n l j}-\epsilon_{F}\right) V_{n l j}^{q}+\sum_{n^{\prime}} \Delta_{n n^{\prime} l j} U_{n^{\prime} l j}^{q}=E_{q l j} V_{n l j}^{q},
\end{gathered}
$$

where $\epsilon_{F}$ is the Fermi energy. The eigenvalues $E_{q l j}$ of these equations are the energies of the quasiparticle states, while the $U$ and $V$ eigenvectors are the occupation amplitudes on the single-particle states. 
The matrix elements of the pairing field, $\Delta_{n n^{\prime} l j}$, can be expressed as

$$
\Delta_{n n^{\prime} l j}=-<n n^{\prime} l j ; J=0|v| \Phi>
$$

where

$$
\left|\Phi>=\sum_{n n^{\prime} l j} \frac{(2 j+1)}{2}\left(\sum_{q} U_{n l j}^{q} V_{n^{\prime} l j}^{q}\right)\right| n n^{\prime} l j ; J=0>
$$

is the pairing density, which is related to the abnormal density as discussed in ref. [6].

The HFB equations are solved self-consistently. Each set of solutions $(U, V)$ determines a new pair density $\Phi$ and new matrix elements of the pairing field, which are then used in the next iteration. This scheme is continued until a converged set of solutions has been obtained. The two particles in Eq. (1) can be in states with a different number of nodes, while in the BCS approximation they are assumed to be in time reversed states $\left(n=n^{\prime}\right)$. The condition $n=n^{\prime}$ is in general too restrictive for an accurate description of localization effects and of the coupling of bound and continuum states [2,66,7].

\section{A. $\mathrm{S}=0$ pairing}

We shall carry out the calculation making use of a Gogny force [8]. In this case, the strongest part of the interaction $v$ is the attractive $S=0$ component. The repulsive $S=1$ component is much weaker and, within the present context, we shall ignore it. The spatial part of the $S=0$ component of the two-particle wavefunction (11) is

$$
\psi_{n n^{\prime} l j}\left(\vec{r}_{1}, \vec{r}_{2}\right)=<\vec{r}_{1}, \vec{r}_{2} \mid n n^{\prime} l j ; J=0>_{S=0}=\frac{1}{4 \pi} \phi_{n l j}\left(r_{1}\right) \phi_{n^{\prime} l j}\left(r_{2}\right) P_{l}\left(\cos \left(\theta_{21}\right)\right),
$$

where $\theta_{21}$ is the angle between $\vec{r}_{1}$ and $\vec{r}_{2}$. Inserting this expression into the $\left(\vec{r}_{1}, \vec{r}_{2}\right)$ representation of the function defined in Eq. (5) we obtain the pairing density

$$
\Phi_{S=0}\left(\vec{r}_{1}, \vec{r}_{2}\right)=\sum_{n n^{\prime} l j}\left(\sum_{q} U_{n l j}^{q} V_{n^{\prime} l j}^{q}\right) \frac{2 j+1}{8 \pi} \phi_{n l j}\left(r_{1}\right) \phi_{n^{\prime} l j}\left(r_{2}\right) P_{l}\left(\cos \left(\theta_{21}\right)\right) .
$$

This quantity is symmetric in the positions of the two particles, whereas the spin part, $\mid S=0>$, is antisymmetric. Equation (17) describes a correlated two-particle state, the 
so-called Cooper pair wavefunction. Because of the $U, V$ factors, this wavefunction receives contributions essentially only from single-particle states around the Fermi surface.

The pairing field is defined as product $\Delta\left(\vec{r}_{1}, \vec{r}_{2}\right)=-v\left(\left|\vec{r}_{1}-\vec{r}_{2}\right|\right) \Phi_{S=0}\left(\vec{r}_{1}, \vec{r}_{2}\right)$. The pairing matrix elements are calculated, (cf. Eq. (4)), by projecting this field onto the two-particle wavefunctions (6). For later discussion we introduce the Fourier transforms of the pairing field $\Delta$ and of the two-particle wavefunctions defined in Eq.(6),

$$
\begin{gathered}
\Delta(\vec{k}, \vec{R})=\int d^{3} r_{12} \Delta\left(\vec{r}_{1}, \vec{r}_{2}\right) e^{-i \vec{k} \cdot \vec{r}_{12}} \\
\psi_{n n^{\prime} l j}(\vec{k}, \vec{R})=\int d^{3} r_{12} \psi_{n n^{\prime} l j}\left(\vec{r}_{1}, \vec{r}_{2}\right) e^{-i \vec{k} \cdot \vec{r}_{12}},
\end{gathered}
$$

where $\vec{r}_{12}$ is the relative distance of the two neutrons and $\vec{R}$ is the center of mass of the pair. The pairing matrix elements can then be written

$$
\Delta_{n n^{\prime} l j}=\frac{1}{(2 \pi)^{3}} \int d^{3} k \int d^{3} R \Delta(\vec{k}, \vec{R}) \psi_{n n^{\prime} l j}(\vec{k}, \vec{R})
$$

\section{B. Local Density Approximation}

Setting the nuclear potential $U$ equal to zero, and allowing the radius $R_{b}$ of the WignerSeitz cell go to infinity for a given value of the Fermi energy $\epsilon_{F}$, one recovers the results valid for uniform neutron matter. In fact, for $R_{b} \rightarrow \infty$ one can substitute $\phi_{n l j}$ by $\left(2 k^{2} / R_{b}\right)^{1 / 2} j_{l}(k r)$, and the sums over $n, n^{\prime}$ by $\left(R_{b} / \pi\right)^{2} \int d k \int d k^{\prime}$. In this limit, the matrix

element of the gap defined in Eq.(四) becomes diagonal in $k$ and $k^{\prime}$, and the HFB equations reduce to those of the BCS theory. The pairing density of Eq. (5) has only one spin component in this case, namely the $S=0$, and it is given by

$$
\Phi_{\text {unif }}\left(r_{12}\right)=\int \frac{d^{3} k}{(2 \pi)^{3}} U_{k} V_{k} e^{i \vec{k} \cdot \vec{r}_{12}}
$$

As expected, the Cooper-pair wavefunction depends, in this limit, only on the relative distance $r_{12}$ of the two neutrons. This is also the case for the associated pairing field 


$$
\Delta_{u n i f}\left(r_{12}\right)=-v\left(r_{12}\right) \int \frac{d^{3} k}{(2 \pi)^{3}} U_{k} V_{k} e^{i \vec{k} \cdot \vec{r}_{12}}
$$

The Fourier transform of this quantity is

$$
\Delta_{u n i f}\left(k, \epsilon_{F}\right)=-\int \frac{d^{3} k^{\prime}}{(2 \pi)^{3}} \frac{\Delta_{u n i f}\left(k^{\prime}, \epsilon_{F}\right)}{2 E_{k^{\prime}}} v\left(\left|\vec{k}-\vec{k}^{\prime}\right|\right) .
$$

In this expression we have explicitly indicated the dependence of the pairing gap on the Fermi energy, and the BCS relation $U_{k} V_{k}=\Delta(k) / 2 E_{k}$ has been used, the quasiparticle energy $E_{k}$ being

$$
E_{k}=\sqrt{\left(\hbar^{2} k^{2} / 2 m-\epsilon_{F}\right)^{2}+\Delta_{u n i f}^{2}\left(k, \epsilon_{F}\right)}
$$

The LDA to the pairing gap is obtained (cf. refs. 9, 10]; cf. also [11]) by replacing $\epsilon_{F}$ in Eqs. (13) and (14) by the local Fermi energy $\epsilon_{F}(R)=\epsilon_{F}-U(R)$. The corresponding local Fermi momentum is given by $\hbar^{2} k_{F}^{2}(R)=2 m \epsilon_{F}(R)$. In this way one obtains a spacedependent pairing gap $\Delta_{L D A}(k, R)=\Delta_{u n i f}\left(k, \epsilon_{F}(R)\right)$ and a space dependent quasiparticle energy,

$$
E_{k}(R)=\sqrt{\left(\hbar^{2} k^{2} / 2 m-\epsilon_{F}(R)\right)^{2}+\Delta_{u n i f}^{2}\left(k, \epsilon_{F}(R)\right)}
$$

First- and second-order corrections in powers of $\hbar$ to the LDA have also been studied [12].

\section{COMPARISON OF HFB AND LDA RESULTS}

In the following we present calculations of the quantum and LDA gaps $\Delta(\vec{k}, \vec{R})$ for a Wigner-Seitz cell of $29 \mathrm{fm}$, and a Fermi energy $\epsilon_{F}=13.5 \mathrm{MeV}$ corresponding to ${ }_{50}^{1800} \mathrm{Sn}$ and typical of the situation encountered at the density of about $3 \times 10^{13} \mathrm{~g} \mathrm{~cm}^{-3}$ ( or $\rho \approx 0.02 \mathrm{n}$ $\mathrm{fm}^{-3}$ ) in the "inner crust" of a neutron star. As for the interaction $v$, we adopt the Gogny force in the ${ }^{1} S_{0}$ channel [8]. In the HFB calculations, we have performed the self-consistent calculation only for the pairing field, while instead of the Hartree-Fock field we have used a Woods-Saxon potential, without introducing an effective mass. The adopted Wood-Saxon potential (cf. Fig. 1) has a depth $V_{o}=-31 \mathrm{MeV}$, a radius $R=7.5 \mathrm{fm}$ and a diffusivity 
$a=0.9 \mathrm{fm}$. These values are chosen in order to reproduce the neutron density in the cell, calculated in ref. [13]. The resulting neutron density is equal to about $\rho_{\text {int }}=0.1 \mathrm{n} \mathrm{fm}^{-3}$ in the interior of the nucleus $(\mathrm{R}<5 \mathrm{fm})$, and then drops to about $\rho_{\text {ext }}=0.018 \mathrm{n} \mathrm{fm}^{-3}$ for 11 $\mathrm{fm}<R<27 \mathrm{fm}$, until it goes to zero close to the boundary of the cell. We have included the single-particle levels up to $100 \mathrm{MeV}$, controlling the convergence of the results. We have also checked that in the absence of the Woods-Saxon potential, we obtain the pairing gap and specific heat of neutron matter at the same Fermi energy. The pairing gap in neutron matter $\Delta_{\text {unif }}\left(k, \epsilon_{F}\right)$, calculated at $k=k_{F}$, is shown in Fig. 2 as a function of $\epsilon_{F}$. In Fig. 2 we also show the value of the local Fermi energy at three points in the Wigner cell.

In neutron matter one can write $\Phi_{\text {unif }} \sim \sin \left(k_{F} r_{12}\right) / k_{F} r_{12}$ as one can see from Eq. (11) taking into account the fact that the product $U_{k} V_{k}$ is peaked at the Fermi energy, so that it oscillates with a wavelength given by $\lambda \approx 2 \pi / k_{F}$. These oscillations are damped over a scale which is approximately determined by the coherence length $\xi=\hbar^{2} k_{F} / m \pi \Delta$ [14, 15]. The pairing gap calculated with the Gogny interaction in neutron matter at the Fermi energy $\epsilon_{F}$ $=13.5 \mathrm{MeV}$ is about $3.5 \mathrm{MeV}$ (cf. Fig. 2), so that one obtain $\xi=3.2 \mathrm{fm}$.

In Fig. 3 we show instead the square of the Cooper pair wavefunctions calculated with HFB in the presence of the nucleus at three values of $R(R=3 \mathrm{fm}, 8 \mathrm{fm}$ and $15 \mathrm{fm})$, as a function of $r_{12}$. At large values of $R$, well outside the nucleus (cf. Fig. 3(c)), the wavefunction is essentially the same as calculated in uniform neutron matter at the same Fermi energy, $\epsilon_{F}=13.5 \mathrm{MeV}$. The radius of the Cooper pair, given by the mean square relative distance, $\left\langle r_{12}^{2}>^{1 / 2}\right.$, is about $4 \mathrm{fm}$, to be compared with the coherence length of 3.2 $\mathrm{MeV}$ reported above. Inside the nucleus, at $R=3 \mathrm{fm}$ (cf. Fig. 3(a)), or on the surface, at $R=8 \mathrm{fm}$ (cf. Fig. $3(\mathrm{~b})$ ), the wavefunction feels the presence of the nucleus, but its main features can still be understood in the same way, using the local values of the Fermi energy and of the pairing field. In this case the wavefunction shows some dependence also on the angle $\theta$ between $\vec{R}$ and $\vec{r}_{12}$ (especially for large values of $r_{12}$ ), and we show the results for $\theta=45^{\circ}$. The local Fermi energy decreases going from inside to outside the nucleus (cf. Fig. 2): it is equal to about $44.5 \mathrm{MeV}$ at $R=3 \mathrm{fm}$, instead of about $25 \mathrm{MeV}$ at $R=8 \mathrm{fm}$ or 
13.5 $\mathrm{MeV}$ at $R=15 \mathrm{fm}$. Therefore the local wavelength $2 \pi / k_{F}(R)$ of the Cooper pair is much shorter inside than outside the nucleus, as can be seen comparing Fig. 3(a) with Fig. 3(b) or Fig. 3(c). Moreover, the pairing gap $\Delta_{L D A}\left(k, \epsilon_{F}(R)\right)$ calculated at the local Fermi momentum $k_{F}(R)$, denoted by $\Delta\left(k_{F}(R)\right)$, is much smaller inside the nucleus than on the surface or outside, (cf. Fig. 2 and Fig. 4(b)), so that the coherence length is larger at $R=$ $3 \mathrm{fm}$, because $k_{F}$ is larger, and $\Delta$ is smaller. Using the values of $\Delta\left(k_{F}(R)\right)(1.6 \mathrm{MeV}$ at $R=$ $3 \mathrm{fm}$ and $3.5 \mathrm{MeV}$ at $R=8 \mathrm{fm}$, cf. Fig. 2), one obtains $\xi=12 \mathrm{fm}$ at $R=3 \mathrm{fm}$ and $\xi=4.1$ $\mathrm{fm}$ at $R=8 \mathrm{fm}$. These values can be compared to the calculated r.m.s. values of $10.3 \mathrm{fm}$ (at $R=3 \mathrm{fm}$ ) and of $3.8 \mathrm{fm}$ (at $R=8 \mathrm{fm}$ ).

The pairing gaps $\Delta(k, R)$ and $\Delta_{L D A}(k, R)$ are presented in Fig. 4(a) and Fig. 4(b) for different values of $k$ as a function of $R$. The value of $\Delta(\vec{k}, \vec{R})$ depends on the angle between $\vec{k}$ and $\vec{R}$, but we have found that this dependence is very small in the present case. The overall dependence on $k$ and $R$ is similar in the two calculations, but the HFB gap shows a smoother spatial variation than the LDA result. This is due to the rather large extension of the Cooper pairs, as discussed above and shown in Fig. 3, leading to important proximity effects, so that the difference in the gap between the interior of the nucleus and the outer part is smeared out. In the semiclassical case, we also show the values of the gap calculated at the local Fermi momentum as a function of $R$. It is seen that $\Delta_{L D A}\left(k_{F}(R)\right)$ is equal to about $1.6 \mathrm{MeV}$ and $3.5 \mathrm{MeV}$ inside and outside the nucleus respectively, as already discussed above.

\section{THE SPECIFIC HEAT}

A quantity playing an important role in the study of neutron stars, is the specific heat of the superfluid phase of the inner crust (cf. e.g. refs. [3], [16], [17]). This quantity is defined as

$$
C_{v}=\frac{1}{V} \frac{\partial<E>}{\partial T}
$$


where $V$ is the volume of the Wigner-Seitz cell. In the framework of our mean-field theory, the energy of the system is simply obtained summing over the quasiparticle states $q$ :

$$
<E>=\sum_{q} n_{q} E_{q}
$$

the quantity $n_{q}=\left(1+e^{\left(E_{q} / T\right)}\right)^{-1}$ being the occupation number of the quasiparticle state $q$.

The LDA result is obtained [16] taking the derivative of the quantity

$$
<E>_{L D A}=\int \frac{d^{3} k d^{3} R}{(2 \pi)^{3}} n_{k}(R) E_{k}(R)
$$

which is the semiclassical counterpart of Eq. (17). In this equation, the occupation factor $n_{k}(R)$ is given by $n_{k}(R)=\left(1+e^{\left(E_{k}(R) / T\right)}\right)^{-1}$, where $E_{k}(R)$ is given by Eq. (15).

In Fig. 5 we compare the specific heat calculated in HFB and in LDA. The semiclassical approximation grossly overestimates the specific heat in the presence of the nuclear potential at low temperatures, while it tends to the quantum result at high temperatures.

In order to better understand the main source of this discrepancy, it is useful to write explicitly the expressions for $C_{v}$ at low temperatures, when $\Delta \gg T$. It is also convenient to use the BCS approximation, which in the present case produces a value for the specific heat that is close to the $\mathrm{HFB}$ result. Using the fact that in BCS $n=n^{\prime}$, one can simply write $\Delta_{n l j}$ and $\psi_{n l j}$ instead of $\Delta_{n n^{\prime} l j}$ and $\psi_{n n^{\prime} l j}$, and one obtains [18]

$$
C_{v}=\frac{1}{V} \frac{\partial}{\partial T} \sum_{n l j} \Delta_{n l j} e^{-\left(\epsilon_{n l j}-\epsilon_{F}\right)^{2} / 2 T \Delta_{n l j}} e^{-\Delta_{n l j} / T}
$$

In the LDA we obtain instead for $\Delta \gg T$,

$$
C_{v, L D A}=\frac{1}{V} \frac{\partial}{\partial T} \int \frac{d^{3} k d^{3} R}{(2 \pi)^{3}} \Delta_{L D A}(k, R) e^{-\left(\hbar^{2} k^{2} / 2 m-\epsilon_{F}(R)\right)^{2} /\left(2 T \Delta_{L D A}(k, R)\right)} e^{-\Delta_{L D A}(k, R) / T}
$$

where we have used the relation given in Eq. (15).

For small temperatures, the dominant contributions to the semiclassical specific heat come from the regions of the phase space $(k, R)$ close to the line defined by the local Fermi momentum $k_{F}(R)$. As shown in Fig. 4(b), there is a clear difference in the values of $\Delta\left(k_{F}(R)\right)$ inside and outside the nucleus, and the integral in Eq. (20) roughly separates in 
two contributions. Although the associated volume is small, the contribution from the inner region is very large, because the local values of the gap are small and are heavily weighted by the exponential factors. If one uses in Eq. (20) the HFB value of $\Delta(k, R)$ instead of the semiclassical values, one obtains an improvement compared the LDA, but the specific heat calculated in this way is still far from the quantum result. Considering instead the quantum expression (19) for the specific heat, and going back to Eq. (10), we observe that the value of the gap for a given state is obtained averaging the value of $\Delta(k, R)$ over $\psi_{n l j}(k, R)$. The main contribution to the specific heat comes from states close to Fermi energy, which are very delocalized, and are rather close to plane waves. On the contrary, Eq. (20) uses the local value of the gap. This suggests to substitute the weighting factor $e^{-\Delta_{L D A}(k, R) / T}$ in Eq. (20) by $e^{-<\Delta_{L D A}(k, R)>/ T}$, where

$$
<\Delta_{L D A}(k, R)>=\int d^{3} R^{\prime} d^{3} k^{\prime} \Delta_{L D A}\left(k^{\prime}, R^{\prime}\right) \psi_{\epsilon}\left(\vec{k}^{\prime}, R^{\prime}\right)
$$

is the average of the semiclassical gap over the density associated with a single-particle state $\phi_{\epsilon}$ of energy close to $\epsilon=\hbar^{2} k^{2} / 2 m+U(R)$. In the present case, it is sensible to approximate the wavefunctions close to the (positive) Fermi energy by plane waves $e^{i \vec{k}_{L}\left(R^{\prime}\right) \cdot \vec{R}^{\prime}} / \sqrt{V}$, so that

$$
\psi_{\epsilon}\left(\vec{k}^{\prime}, R^{\prime}\right) \approx \frac{1}{V} \delta\left(\vec{k}^{\prime}-\vec{k}_{L}\left(R^{\prime}\right)\right)
$$

where the local momentum $k_{L}\left(R^{\prime}\right)$ is defined by

$$
\frac{\hbar^{2}}{2 m} k_{L}^{2}\left(R^{\prime}\right)+U\left(R^{\prime}\right)=\epsilon,
$$

and

$$
<\Delta_{L D A}(k, R)>=\frac{1}{V} \int d^{3} R^{\prime} \Delta_{L D A}\left(k_{L}\left(R^{\prime}\right), R^{\prime}\right)
$$

Using the occupation factor $e^{-<\Delta_{L D A}>/ T}$ in Eq. (20), one obtains a specific heat which is very close to the exact result at all temperatures, as illustrated in Fig. 5.

We conclude that the local density approximation to the pairing phenomenon overestimates the spatial dependence of the gap, because it does not take into account the 
non-locality of the Cooper pairs, and the associated proximity effects. Particular care has to be taken in estimating the thermal occupation factors, for which the LDA can lead to large errors at low temperatures. However, at least in the present case, it is possible to obtain an accurate approximation to the quantum HFB result by a simple averaging of the semiclassical expression of the pairing gap.

\section{ACKNOWLEDGMENTS}

This work was supported in part (H.E.) by the U.S. Department of Energy, Nuclear Physics Division, under contract No. W-31-109-ENG-38. 


\section{REFERENCES}

[1] D. Pines and M. Ali Alpar, in Neutron Stars, eds. D. Pines, R. Tamagaki, and S. Tsuruta, Addison-Wesley, Reading, Mass. (1992).

[2] F. Barranco, R.A. Broglia, H. Esbensen and E. Vigezzi, Phys. Lett. B390 (1997) 13.

[3] C.J. Pethick and D.G. Ravenhall, Annu. Rev. Nucl. Part. Sci. 45 (1995) 429.

[4] D. Pines and M. Ali Alpar, in The Structure and Evolution of Neutron Stars, eds. D. Pines, R. Tamgaki and S. Tsuruta, Addison-Wesley.

[5] P. Ring and P. Schuck, The Nuclear Many-Body Problem, Springer Verlag (1980).

[6] J. Dobaczewski, H. Flocard and J. Treiner, Nucl. Phys. A422 (1984) 103.

[7] J. Dobaczewski, W. Nazarewicz, T.R. Werner, J.F. Berger, C.R. Chinn and J. Dechargé, Phys. Rev. C53 (1996) 2809.

[8] J.Dechargé and D. Gogny, Phys. Rev. C21 (1980) 1568.

[9] R. Bengtsson and P. Schuck, Phys. Lett. 89B (1980) 321.

[10] R.A. Broglia and A. Winther, Phys.Lett. 124B (1983) 11.

[11] L.N. Oliveira, E.K.U. Gross, and W. Kohn, Phys. Rev. Lett. 60 (1988) 2430.

[12] K. Taruishi and P. Schuck, Z. Phys. A342 (1992) 397.

[13] J. Negele and D. Vautherin, Nucl. Phys. A207 (1973) 298.

[14] P.G. de Gennes, Superconductivity of Metals and Alloys, Addison-Wesley (1989).

[15] V. Weisskopf, Cont. Phys. 22 (1981) 375.

[16] R.A. Broglia, F. de Blasio, G. Lazzari, M. Lazzari, and P. Pizzochero, Phys. Rev D50 (1994) 4781.

[17] F. de Blasio, G. Lazzari, P. Pizzochero and R.A. Broglia, Phys. Rev. D53 (1996) 4226. 
[18] L. Landau and E. Lifschitz, Statistical Mechanics, Part 2, Pergamon Press (1980).

\section{Figure captions}

\section{Fig. 1}

The Woods-Saxon potential $U(R)$ in the Wigner-Seitz cell of radius $R_{b}=29 \mathrm{fm}$ is shown. We also indicate the Fermi energy $\epsilon_{F}=13.5 \mathrm{MeV}$, and the outer neutron density $\rho_{\text {ext }}$.

\section{Fig. 2}

The pairing gap $\Delta_{u n i f}\left(k, \epsilon_{F}\right)$ calculated with the Gogny interaction in uniform neutron matter at the Fermi momentum $k_{F}$ is shown as a function of the Fermi energy. Also indicated by the arrows are the values of the local Fermi energy $\epsilon_{F}(R)$ in the Wigner-Seitz cell at $R=$ $3 \mathrm{fm}, 8 \mathrm{fm}$ and $15 \mathrm{fm}$.

\section{Fig. 3}

The dependence of the square of the Cooper pair wavefunction $\Phi\left(R, r_{12}\right)$ on the relative distance $r_{12}$ is shown for three different values of the center of mass of the Cooper pair in the Wigner-Seitz cell, $R=3 \mathrm{fm}(\mathrm{a}), 8 \mathrm{fm}(\mathrm{b})$ and $15 \mathrm{fm}(\mathrm{c})$.

Fig. 4

The pairing gap $\Delta(k, R)$ is calculated as a function of $R$ in the Wigner cell, using the HFB (a) or the LDA (b) equations. The gap remains constant for $R>12 \mathrm{fm}$. The various curves refer to different values of $k$, namely $k=0.25,0.75,1.25,1.75$ and $2.5 \mathrm{fm}^{-1}$ going from the top to the bottom curve. In figure (b), we also show the value of the gap calculated at local Fermi momentum $k_{F}(R)$ (dashed curve).

Fig. 5 
Specific heat of a Wigner cell containing a nucleus (in units of $\mathrm{MeV} \mathrm{k}^{-1} \mathrm{fm}^{-3}$ ) calculated according to the HFB approximation, to the LDA approximation, and to the LDA corrected using the averaged pairing gap $<\Delta_{L D A}>$ according to Eq. (24). 


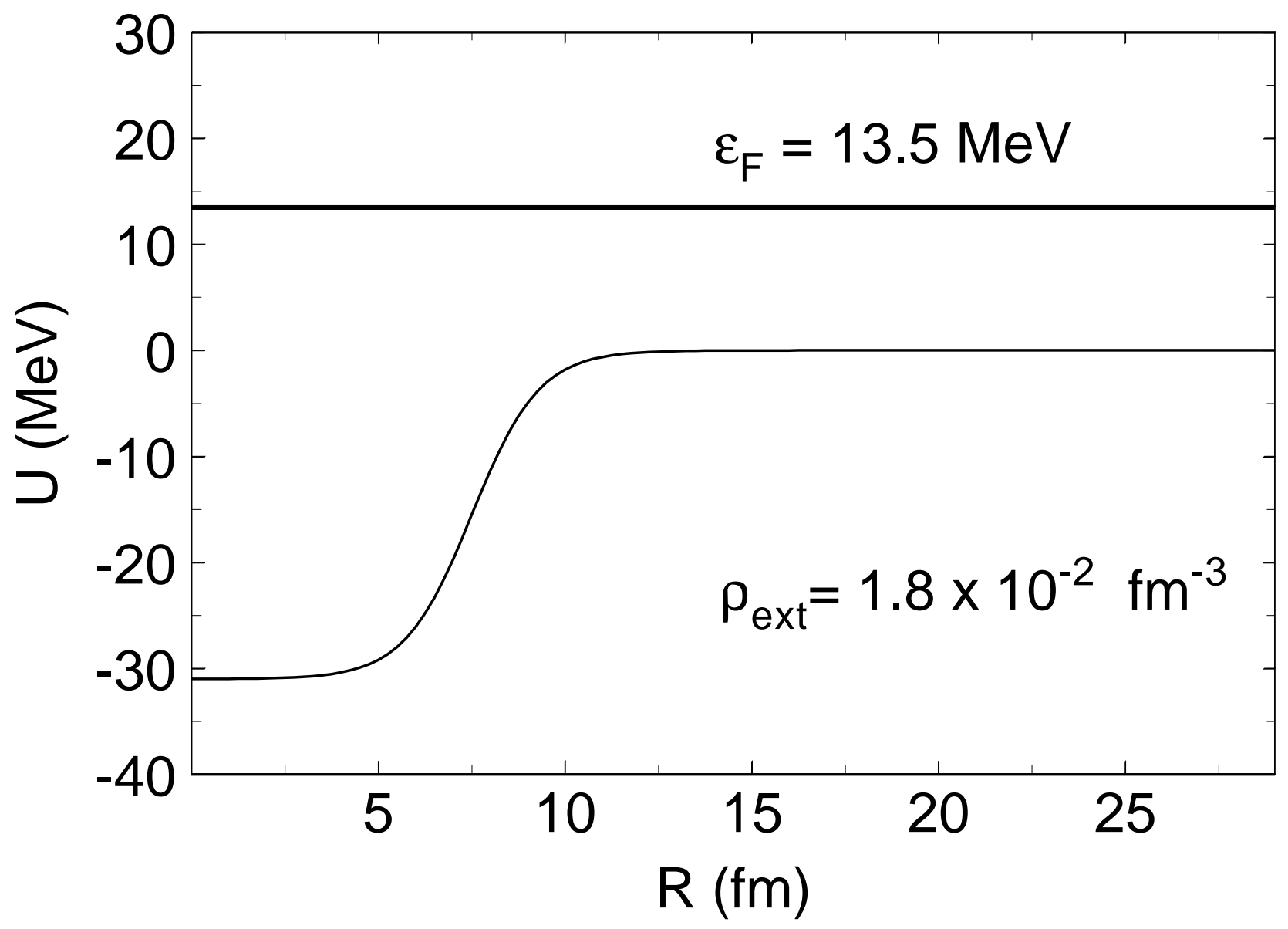




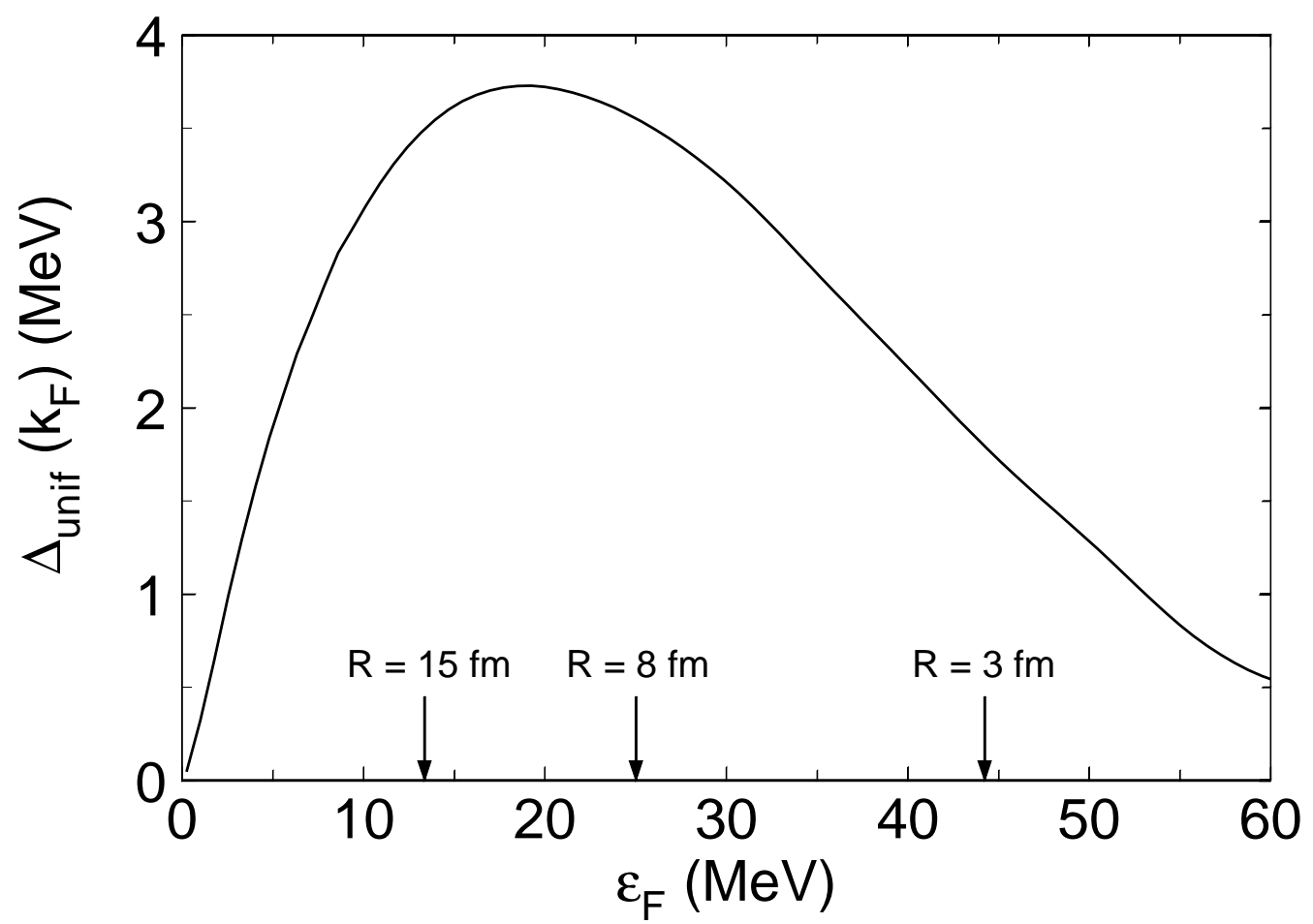




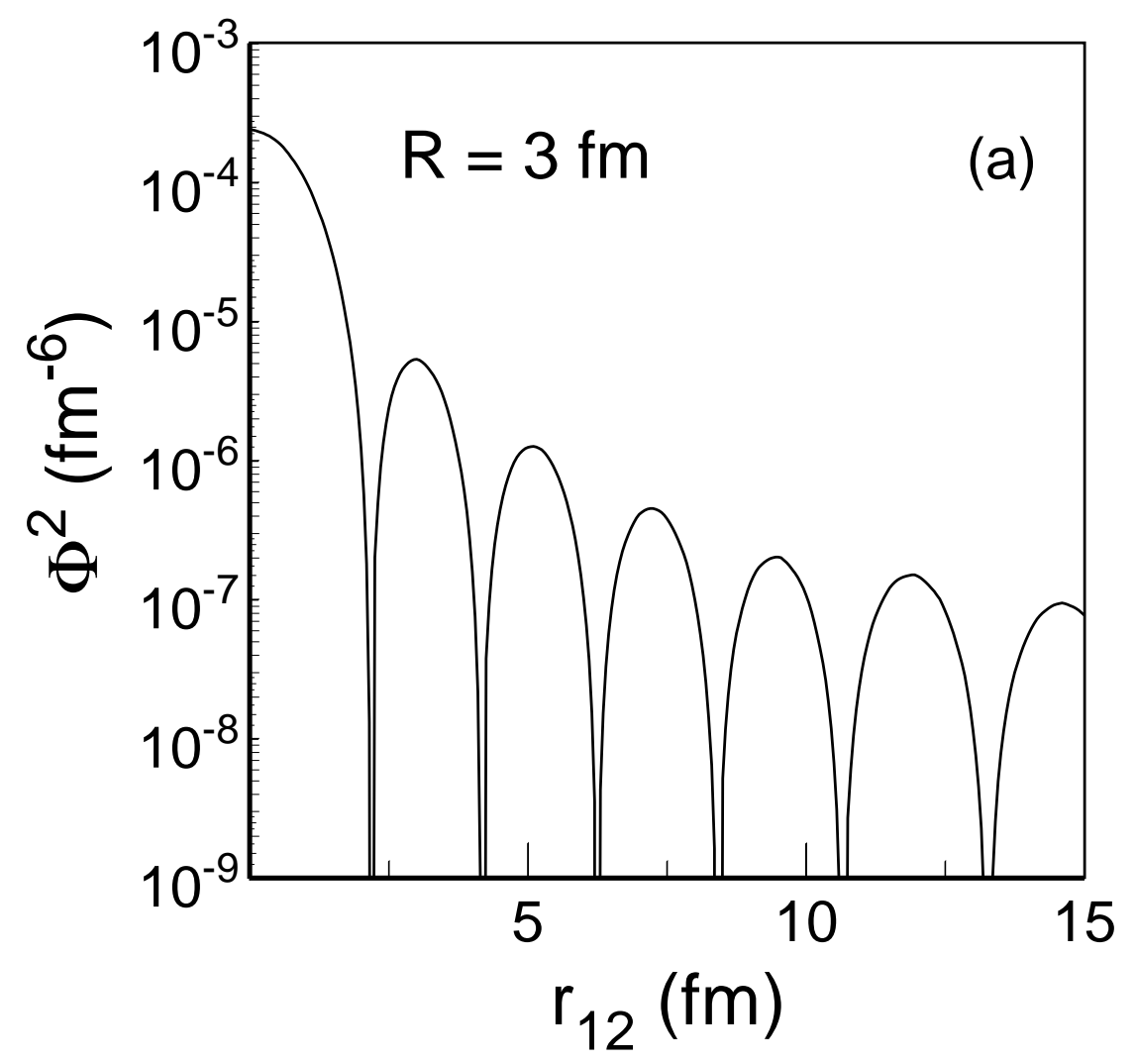




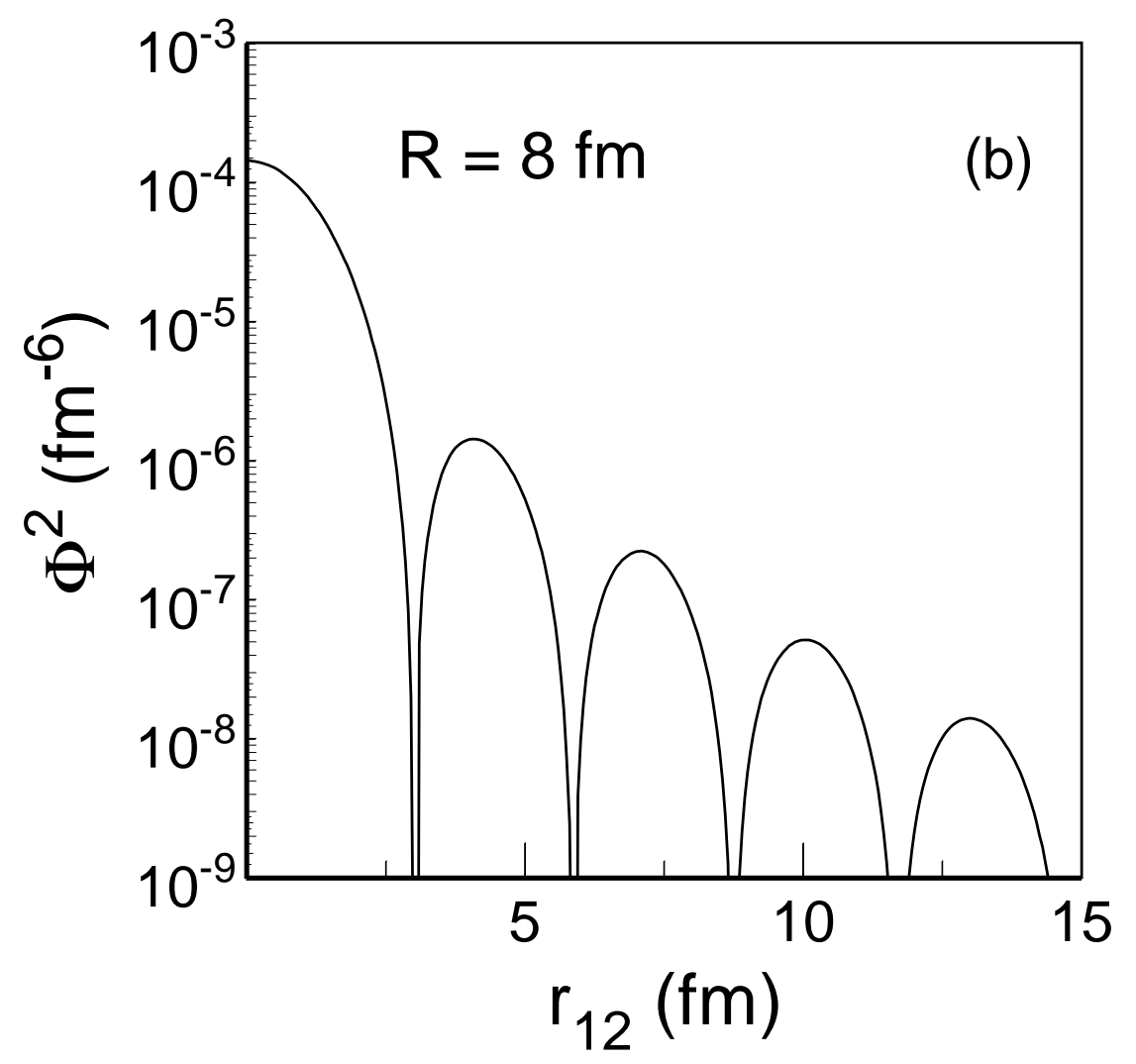




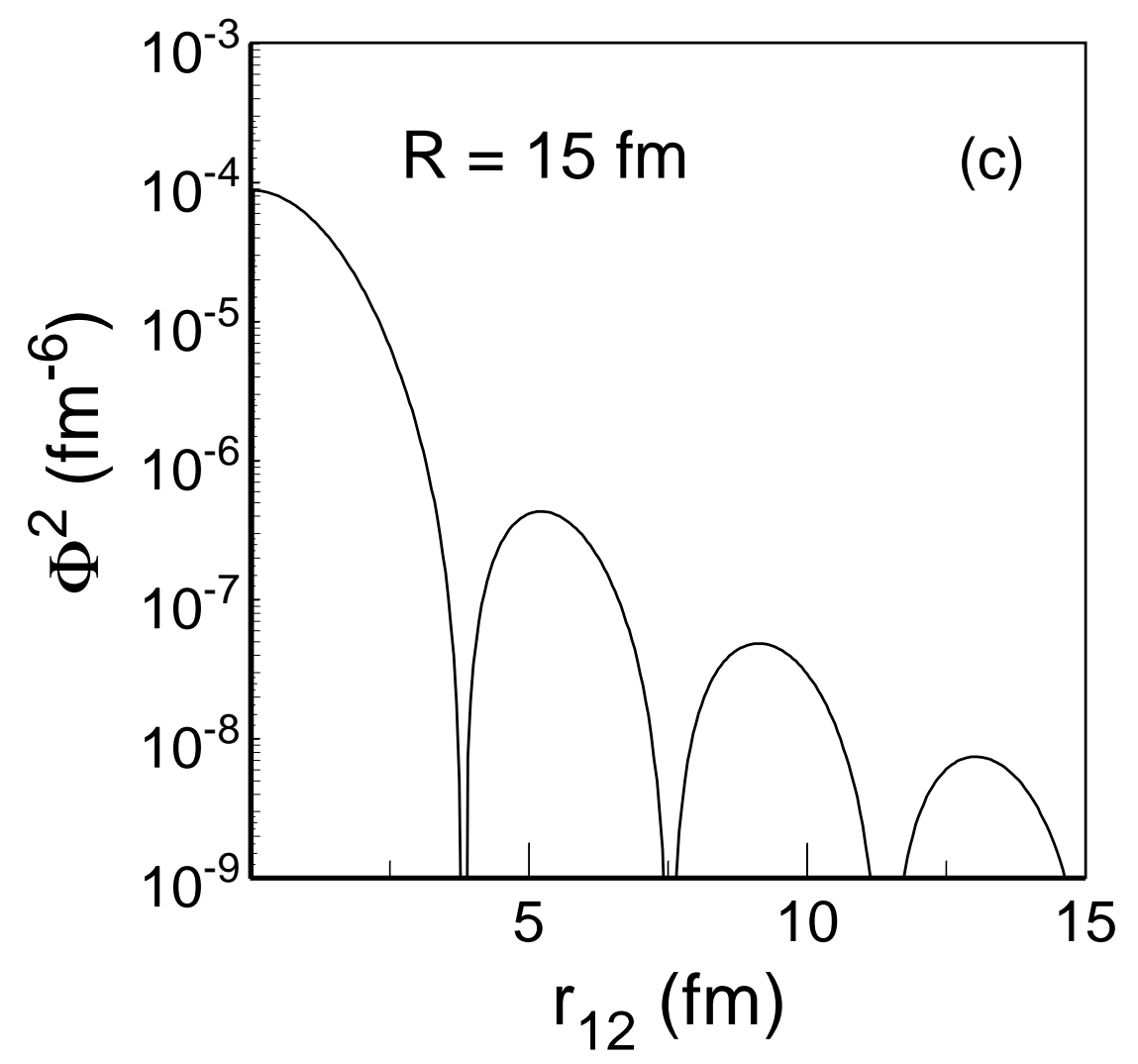




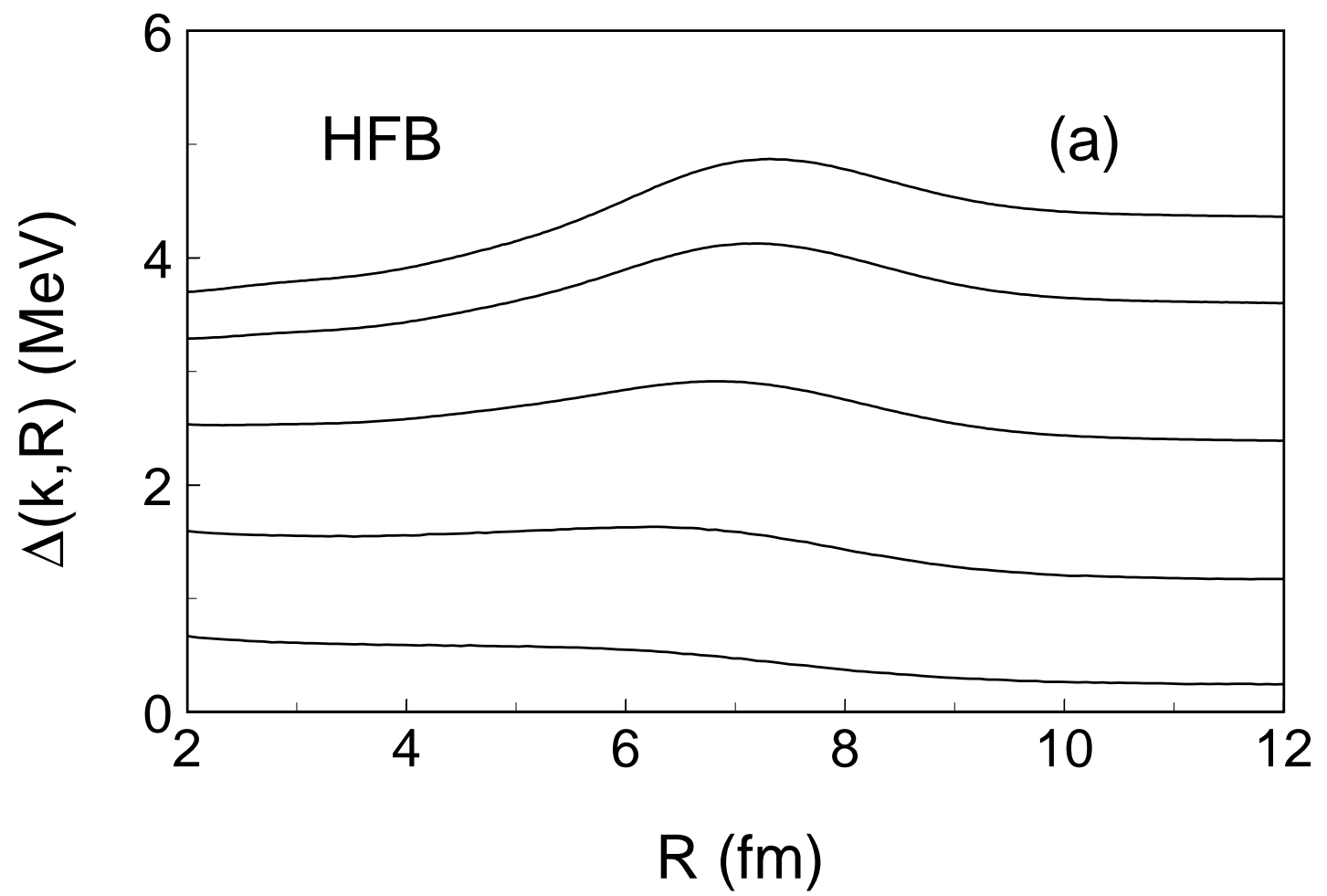




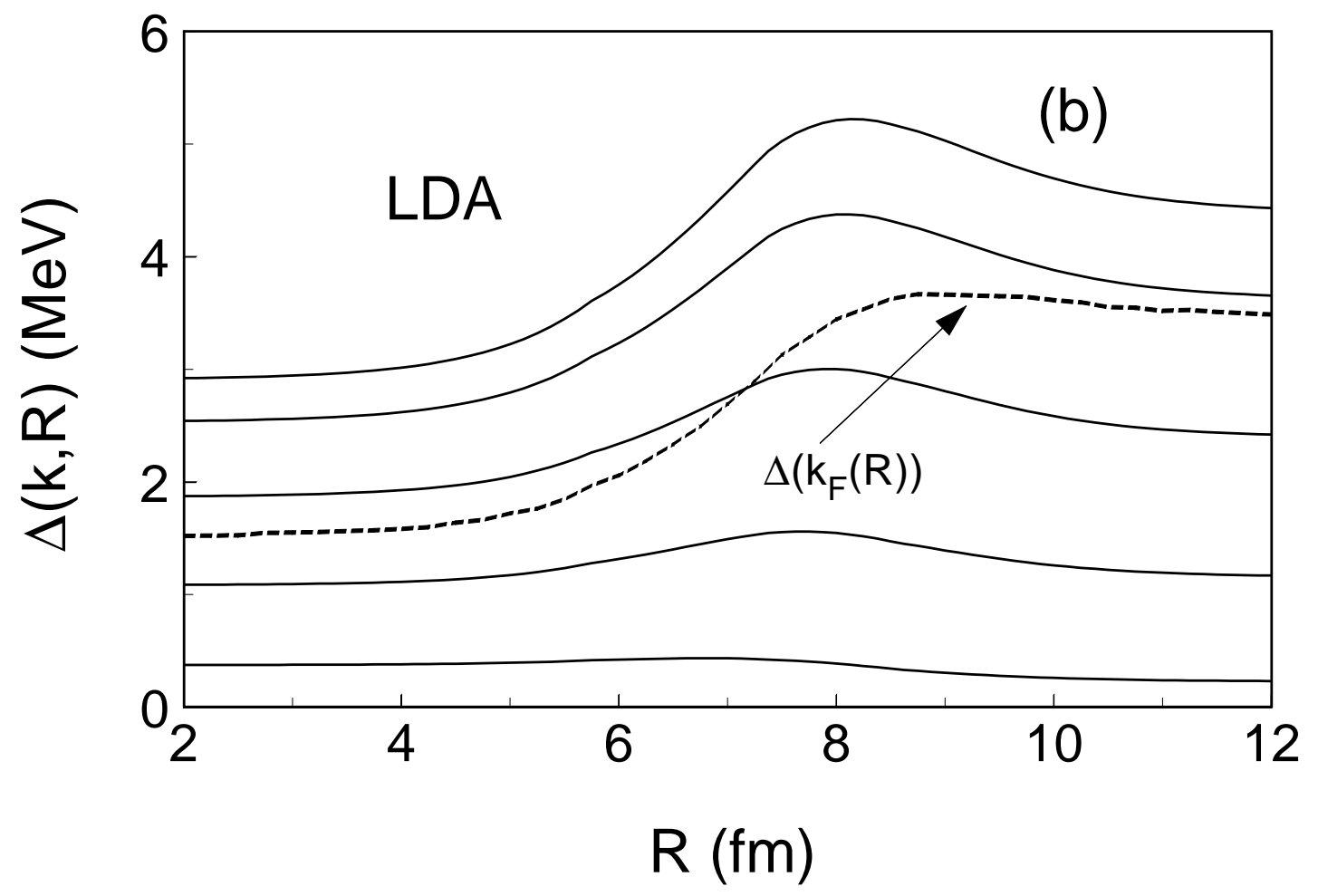




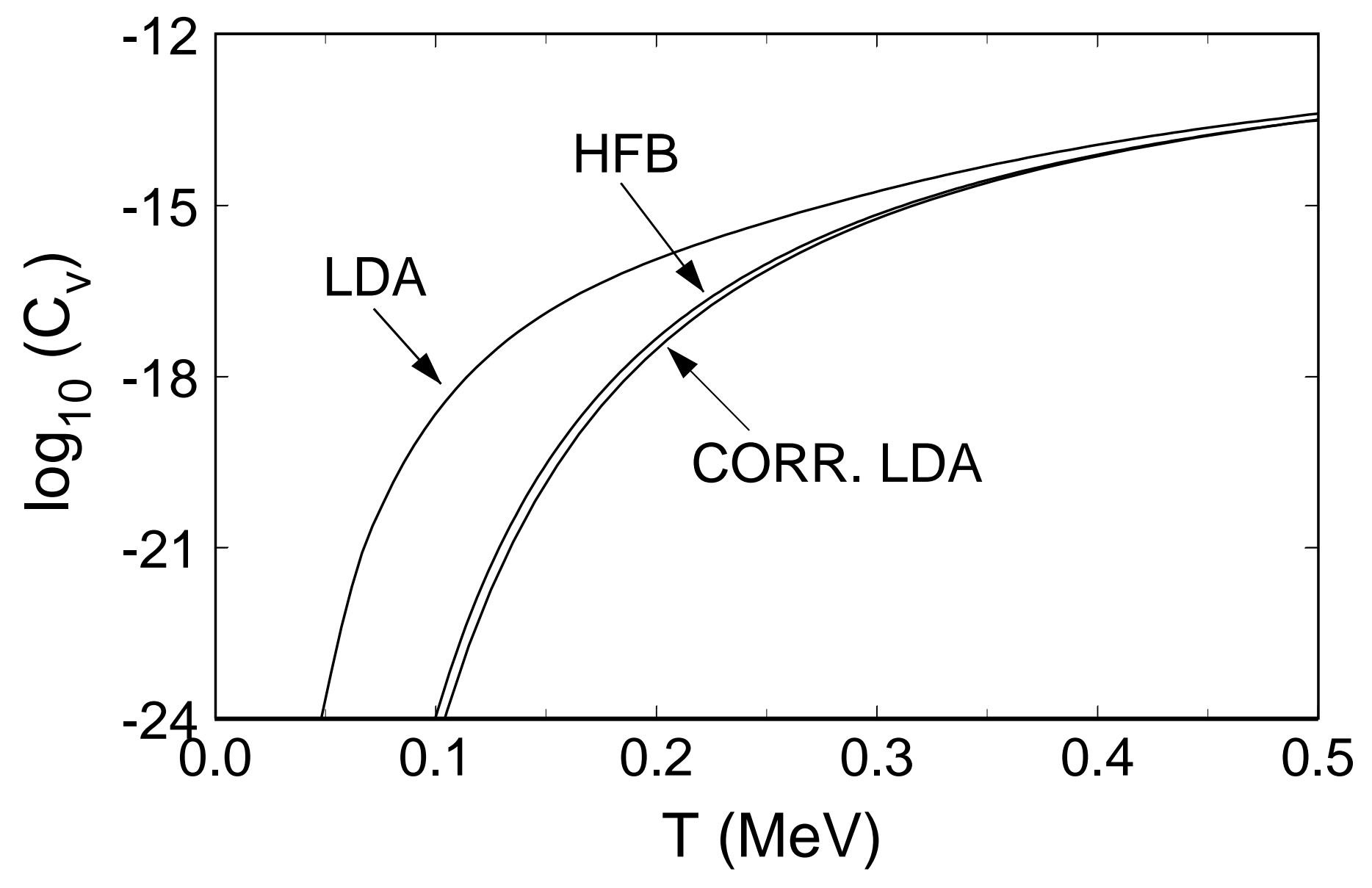

